\title{
Co-Production of Nattokinase and Poly ( $\gamma$-Glutamic Acid) Under Solid-State Fermentation Using Soybean and Rice Husk
}

\author{
Guangjun Nie, Zhubin Zhu, Fang Liu, Zhijie Nie, Yuchao Ye and Wenjin Yue \\ College of Biochemical Engineering; Anhui Polytechnic University; Wuhu - China
}

\begin{abstract}
The aim of this work was to study the co-production of nattokinase and poly ( $\gamma$-glutamic acid) by Bacillus subtilis natto with soybean and rice husk under solid-state fermentation (SSF). The results showed that the size of soybean particle and rice husk significantly improved the co-production of nattokinase and poly (y-glutamic acid), yielding $2503.4 \mathrm{IU} / \mathrm{gs}$ and $320 \mathrm{mg} / \mathrm{gs}$, respectively in the improved culture medium composed of $16.7 \%$ soybean flour and $13.3 \%$ rice husk with $70 \%$ water content. The yields increased by approximate 7-and 2-fold factor relative to their original ones. Thus, the co-production of nattokinase and poly ( $\gamma$-glutamic acid) under SSF could be considered as an efficient method to exploit agro-residues for economical production of some higher-value products.
\end{abstract}

Key words: Co-production, Nattokinase, poly ( $\gamma$-glutamic acid), Rice husk, Solid-state fermentation

\section{INTRODUCTION}

Intravascular thrombosis, which results from blood clot fibrin in arteries, is a bad disease against human health. As compared to the clinical thrombolytic drugs (urokinase and streptokinase), nattokinase (NK) with many advantages such as confirmed efficacy, prolonged effects, cost effectiveness and preventative use is a potent thrombolytic enzyme in vitro and in vivo (Zheng et al. 2005). For example, it has four times fibrinolytic activity over plasmin (Sumi et al. 1992), and the activity can be retained in the blood for more than $3 \mathrm{~h}$ (Fujita et al. 1995). In view of the traits like safe, low cost, and easy oral administration, NK has been considered as an excellent functional food for thrombosis therapy.

Poly $(\gamma$-glutamic acid) or $\gamma$-PGA is a homopolymer of D- and L-glutamic acid units, which is biodegradable, edible and nontoxic toward humans and environment (Shih and Van 2001). Accordingly, it is a promising environmental-friendly biomaterial with broad industrial application in the fields of medicine, foods, plastics, farming and many others (Shih and Van 2001; Wang et al. 2008; Zhu et al. 2010).

Bacillus subtilis has been usually applied to produce NK under submerged fermentation $(\mathrm{SmF})$ (Deepak et al. 2008) and to manufacture $\gamma$-PGA under solid-state fermentation (SSF) (Wang, et al. 2008; Yao et al. 2012; Zeng et al. 2013). This strain has been applied to co-produce multiple biomaterials containing $\gamma$-PGA under SSF (Wang et al. 2008; Yao et al. 2012; Zeng et al. 2013).

There are a large number of agricultural and forestry residues produced world-wide whose disposal is a serious problem in China. However, these could be effectively used in SSF for enzyme production (Jana et al. 2012; Selwal and Selwal 2012; Beniwal et al. 2013; Wang et al. 2013).

*Author for correspondence: join-us@163.com 
Soybean and rice husk are low-cost and abundant in China. Rice husk, in particular, is an excellent solid support for SSF in view of its uniform shape of the particles with fluffy and elastic characteristics. On the other hand, $\gamma$-PGA fermentation broth under $\mathrm{SmF}$ is highly viscous and exhibits non-Newtonian rheology along with a serious barrier against mixing, heat transfer and oxygen supply (Richard and Margaritis 2003). Impurities in the liquid fermentation increase the cost for $\gamma$-PGA purification. As compared to SmF, SSF can not only exploit cheap and easily available raw agro-residues but also offers economical and engineering advantages such as low processing cost, less energy expenditure, simple equipment and facilities (Pandey 2003; Chen et al. 2005). $\gamma$-PGA, an important composition of natto, can promote mineral substance to be utilized. It also can preserve the moisture of solid culture medium and further improve NK production under SSF. Accordingly, it is considered that co-production of NK and $\gamma-$ PGA under SSF should be an interesting work. To our knowledge, the co-production, especially with soybean and rice husk as solid matrix, has not yet been reported. In this work, soybean and rice husk were utilized as solid substrate and support for SSF to co-produce NK and $\gamma$-PGA using Bacillus subtilus natto.

\section{MATERIAL AND METHODS}

\section{Material}

NK-producing strain was obtained from the commercial Bacillus subtilis natto powder and maintained in $4^{\circ} \mathrm{C}$. Casein was purchased from Sinopharm Chemical Reagent Co., Ltd. (SCRC), China. Thrombin, cattle fibrinogen and urase were bought from the National Institute for the Control of Pharmaceutical and Biological Products (NICPBF). Rice husk and Chinese northeast soybean were bought from the local market.

\section{CO-production of NK And $\gamma$-PGA Under SSF}

A dosage of $500 \mu \mathrm{L}$ Bacillus subtilus natto solution was inoculated in $150 \mathrm{~g}$ boiled Chinese soybeans in a plastic case $(\Phi 20 \mathrm{~cm})$. After being mixed evenly, the solid culture medium was covered with three layers of gauzes, then the case was sealed with foodstuff preservative film with six holes $(\Phi 2 \mathrm{~mm})$ and incubated at $37^{\circ} \mathrm{C}$ for $24 \mathrm{~h}$.
Afterwards, the whole contents were preserved at $4^{\circ} \mathrm{C}$ for $24 \mathrm{~h}$.

\section{Extraction of Crude NK And Determination Its Activity}

Sixteen gram fermented matrix was added in 40 $\mathrm{mL}$ normal saline in $250 \mathrm{~mL}$ flask, mixed at 100 $\mathrm{rpm}$ for $1 \mathrm{~h}$ and centrifuged (10,000 rpm, $10 \mathrm{~min})$. The above extraction protocols were carried out two times. The supernatant was collected and defined as crude NK. NK activity was determined using the method of Wei et al. (2007). Briefly, 2.0 $\mathrm{mL} 0.5 \%$ casein solution and $1.0 \mathrm{~mL}$ crude $\mathrm{NK}$ samples were incubated at $30^{\circ} \mathrm{C}$ for $5 \mathrm{~min}$, then the NK sample was mixed with the casein solution uniformly and incubated at $30^{\circ} \mathrm{C}$ for $10 \mathrm{~min}$. Afterwards, $3.0 \mathrm{~mL} 10 \%$ trichloroacetic acid was added in the mixture and incubated for $10 \mathrm{~min}$, then filtered. A control was prepared by mixing $1.0 \mathrm{~mL}$ crude NK sample with $3.0 \mathrm{~mL} 10 \%$ trichloroacetic acid uniformly and then $2.0 \mathrm{~mL}$ $0.5 \%$ casein solution was added in the mixture. Other protocols were the same as the above mentioned. Then $1.0 \mathrm{~mL} \mathrm{NK}$ sample and $1.0 \mathrm{~mL}$ the control were added in two tubes containing 5.0 $\mathrm{mL} 0.55 \mathrm{~mol} / \mathrm{L}$ sodium carbonate and $1.0 \mathrm{~mL}$ phenol, respectively and incubated at $30^{\circ} \mathrm{C}$ for 15 min after mixing well. The absorbance of the sample and the control was read by an ultraviolet spectrophotometer (SP-754, Shanghai Spectrum Instruments Co Ltd, China) at $680 \mathrm{~nm}$, which were defined as $A_{s}$ and $A_{c}$, respectively. NK activity was calculated based on tyrosine calibration curve and its achievable model $\left(\mathrm{NK}\right.$ activity $=\mathrm{K}^{*}\left(\mathrm{~A}_{\mathrm{s}}-\right.$ $\left.A_{c}\right) * N * 6 / 10$, where $K$ was the slope reciprocal of tyrosine calibration curve, $\mathrm{N}$ was the distilled fold). The unit of NK activity was defined as the $\mathrm{NK}$ dosage required for $1.0 \mu \mathrm{g}$ tyrosine formation in $1 \mathrm{~min}$. All the experiments were carried out in three sets.

\section{Extraction of Crude $\gamma$-PGA And Determination Its Yield}

The extraction method in this work was modified based on the method by Bajaj et al.(2008). Twenty gram fermented substrate was added in $80 \mathrm{~mL}$ normal saline in $250 \mathrm{~mL}$ Bunsen beaker and mixed at $30^{\circ} \mathrm{C}$ on a rotary shaker $(200 \mathrm{rpm})$ for $1 \mathrm{~h}$, then the mixed solution was filtered by two-layer gaze. The filtrate was centrifuged at $10000 \mathrm{rpm}$ for 15 min. Clarified supernatants were collected for $\gamma$ PGA purification. 
$\gamma$-PGA was purified by the method reported by Goto and Kuniko(1992). Ten milliliters of supernatant was poured in $40 \mathrm{~mL}$ methanol and kept at $4^{\circ} \mathrm{C}$ for $12 \mathrm{~h}$. Crude $\gamma$-PGA was collected by centrifugation at $10000 \mathrm{rpm}$ and $4^{\circ} \mathrm{C}$ for $30 \mathrm{~min}$ and then it was dissolved in distilled water. Any insoluble impurity was removed by centrifugation. The aqueous $\gamma$-PGA solution was desalted by dialysis (molecular weight cut off 3500) against 1 $\mathrm{L}$ distilled water for $12 \mathrm{~h}$ with water exchange three times. $\gamma$-PGA yield was determined by UV assay according to the reported method previously (Zeng et al. 2012). All the experiments were carried out in three sets.

\section{RESULTS AND DISCUSSION}

\section{Time courses of NK and $\boldsymbol{\gamma}$-PGA production}

Studies on finding the optimal time for NK and $\gamma$ PGA production showed the maximum NK yield, $4.21 \mathrm{U} / \mathrm{g}$ dry substrate (U/gds or U/g) at $24 \mathrm{~h}$ (Fig. 1A). Afterwards, the yield began to decrease. As shown in Figure1B, the $\gamma$-PGA yield exhibited a trend from ascent to descent with the increment of incubation time and the maximum yield was 144 $\mathrm{mg} / \mathrm{g}$ at $36 \mathrm{~h}$. The optimal time of $\gamma$-PGA production was shorter than the previous report, $72 \mathrm{~h}$ (Bajaj et al. 2008).
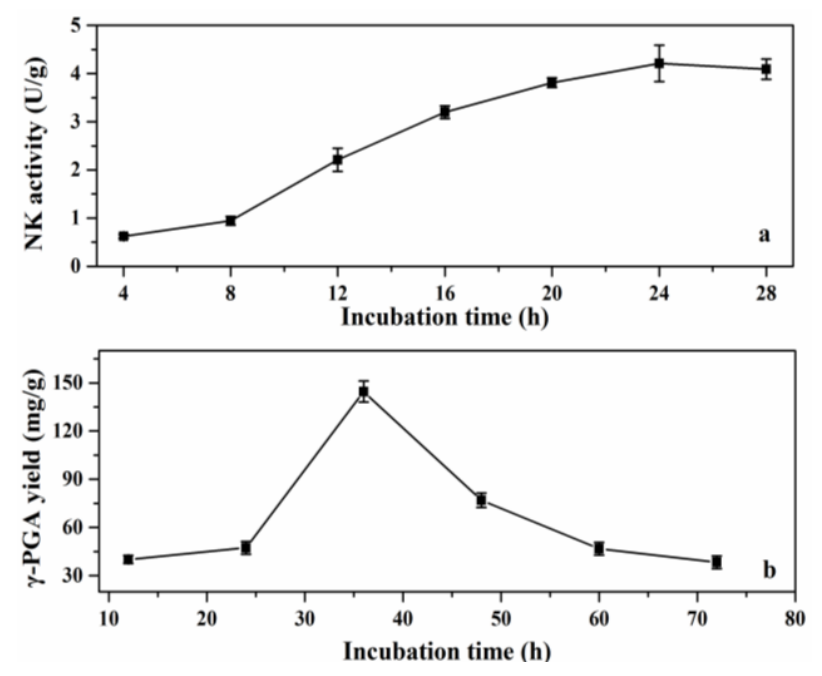

Figure 1 - Schedule for NK (A) and $\gamma$-PGA (B) production.

Effect of particle size of Soybean on NK and $\gamma$ PGA Production

The effect of the particle size of solid substrate (whole bean, quarter bean, and bean flour) on coproduction of NK and $\gamma$-PGA showed that the maximum yields of NK and $\gamma$-PGA were obtained with bean flour as substrate. It was inferred that NK and $\gamma$-PGA production could be enhanced by the small size of substrate particles.
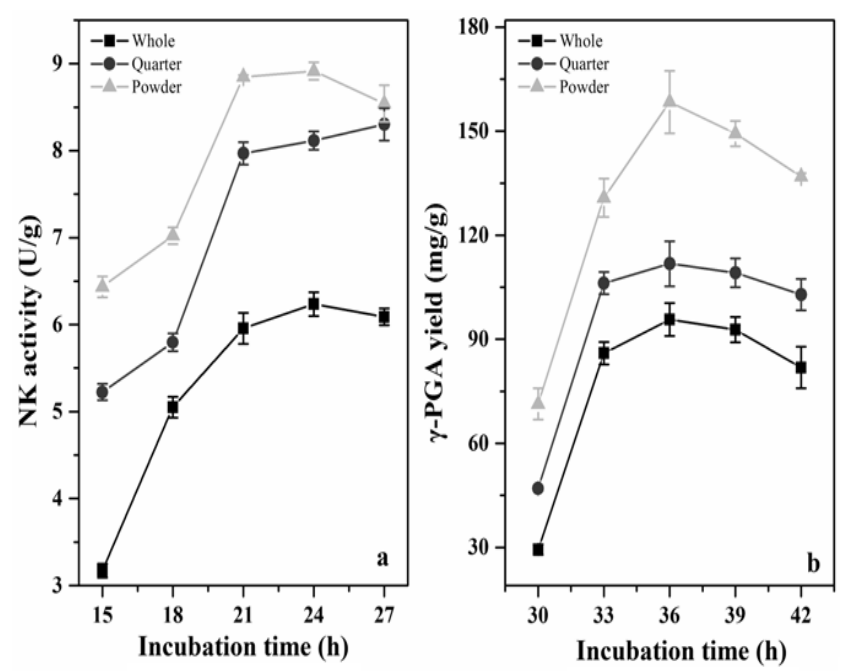

Figure 2 - Effect of substrate particles size on NK (A) and $\gamma$-PGA (B) production.

"Whole" referred to whole soybean as sold substrate under SSF. "Quarter" referred to smaller particle size of soybean residue, which was one quarter, the size of whole soybean. "Powder" referred to soybean flour. Inoculum size and water size were 4 and $70 \%$, respectively. The other conditions were same as described in the "Material and methods" section.

Smaller size of solid substrate particles provides larger surface area. The enlarged area is helpful for mass transfer by enhancing the affinity of substrate to microbial cells. Thus, the substrate containing small size particles gave higher yields of NK and $\gamma$-PGA by meeting the rapid requirement of microbial metabolism for nutrients, particularly at the latter stage of metabolite synthesis. However, the small size effect could also inhibit the metabolite synthesis by blocking air to flow fluently. It was deduced that nutrient rather than oxygen was even more necessary for the microbial metabolism at the latter stage. Nevertheless, the poor oxygen concentration available in solid matrix has a harmful effect on microbial metabolism. Therefore, it is important that a desired balance between particle size and air-flow rate is established in SSF processes.

\section{Effect of Rice Husk on NK And $\gamma$-PGA Production}

The effect of particle size of rice husk on air-flow was studied, the result indicated that rice husk can improve the strain in co-producing $\mathrm{NK}$ and $\gamma$ PGA. The improvement was more prominent for 
NK production than for $\gamma$-PGA production (Fig. $3)$. Figure 4 showed that the NK yield kept on decreasing with the increment of rice husk content. On the contrary, the yield of $\gamma$-PGA kept on increasing until the ratio of rice husk to soybean grew up to 5:4, when the maximum yield (227.6 $\mathrm{mg} / \mathrm{g}$ ) was achieved.
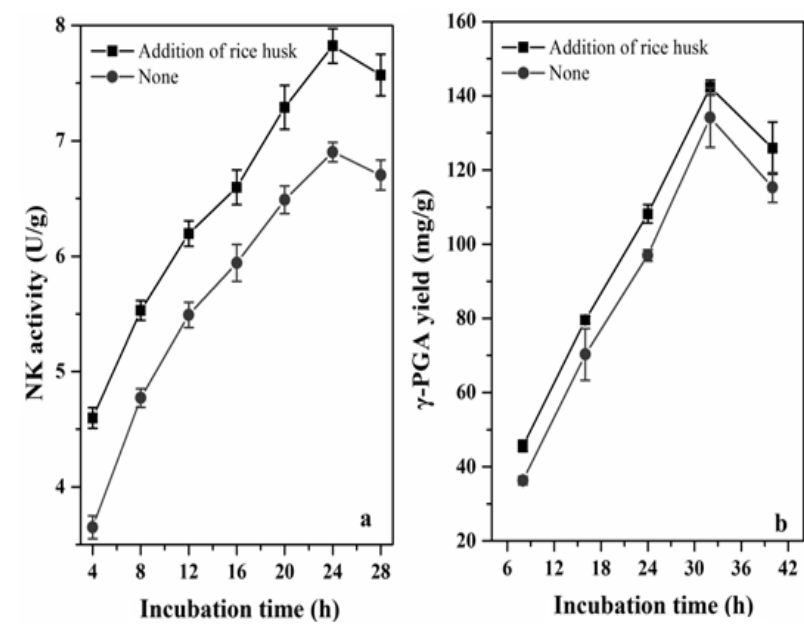

Figure 3 - NK (a) and $\gamma$-PGA (b) production using rice husk.

In this diagram, "Addition of rice husk" refers to direct addition $30 \mathrm{~g}$ rice husk in the solid fermentation medium containing $150 \mathrm{~g}$ soybean flour, "None" refers to the solid fermentation medium without any rice husk. Inoculum size and water size were $4 \%$ and $70 \%$, respectively. The other conditions were seen in the "Material and methods" section.

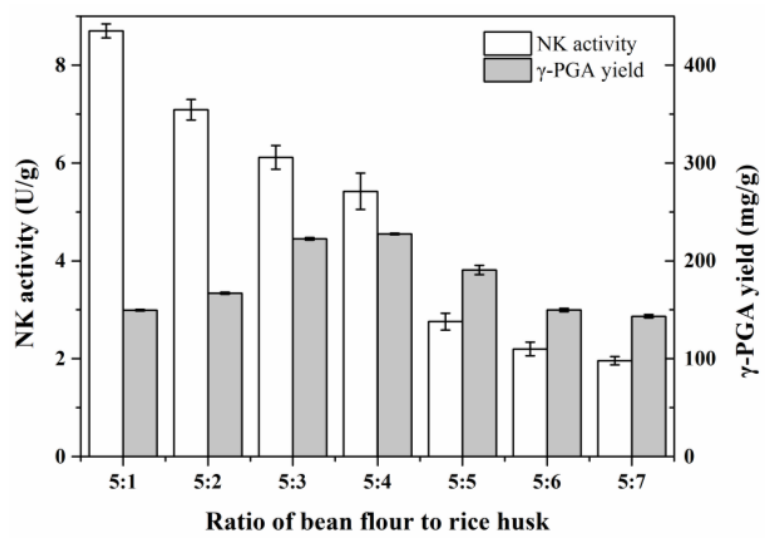

Figure 4 - Effect of the ratio of bean flour to rice husk on $\mathrm{NK}$ and $\gamma$-PGA production.

In this experiment, the solid fermentation medium was composed of $30 \mathrm{~g}$ soybean flour and different amount of rice husk decided by the ratio of bean flour to rice husk. Inoculum size and water size were $4 \%$ and $70 \%$, respectively. NK activity was assayed after the strain was incubated for $24 \mathrm{~h}$, and $\gamma$-PGA yield was determined after the strain was incubated for $36 \mathrm{~h}$. The other conditions were seen in the "Material and methods" section.
Accordingly, it was concluded that the enhancement in air flow could enhance the coproduction of NK and $\gamma$-PGA. In view of its fluffy and elastic nature, rice husk could make the compact culture medium (composed by fine soybean particles) so fluffy that countless new "air-lines" are can be constructed. Besides, rice husk can enlarge the binding area of microbial cell to substrate by making an even and extensive distribution of microorganism in solid culture medium. This not only improved the air permeability and heat dissipation in the solid medium but also speeded up mass transfer of substrate and product. The fermented rice husk could be utilized to improve organic fertilizer in water retention and nutrients (Chen et al. 2005). This was in agreement with the present increasing emphasis on exploiting agro residues. Therefore, rice husk could be regarded as an excellent leavening agent for SSF.

\section{Effects of Water Content and Inoculum Size on NK and $\gamma$-PGA Production}

In view of moisture retention of $\gamma$-PGA, different water contents $(50-90 \%)$ were investigated to find the optimum of moisture level. Figure 5 indicated that the water content at $70 \%$ was the best initial moisture level for NK and $\gamma$-PGA production.

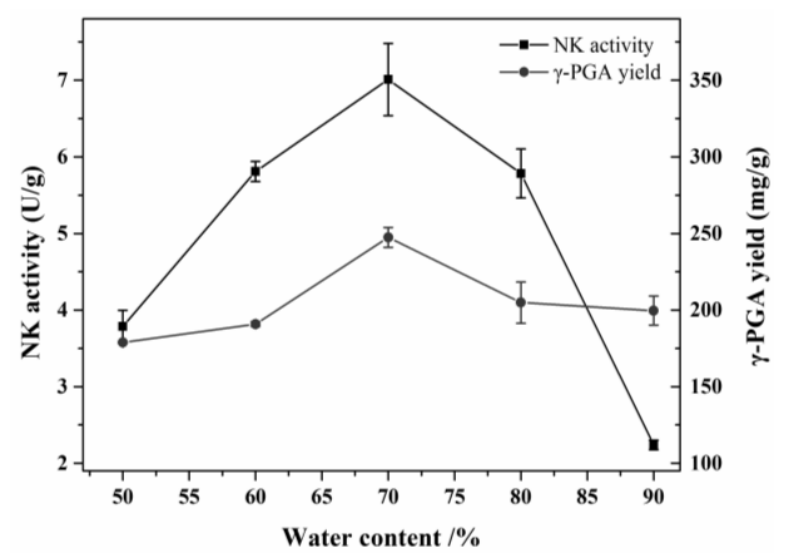

Figure 5 - Effect of the water content on NK and $\gamma$ PGA production.

In this experiment, the solid fermentation medium was composed of $30 \mathrm{~g}$ soybean flour and $18 \mathrm{~g}$ rice husk. Inoculum size was determined as $4 \%$. NK activity was assayed after the strain was incubated for $24 \mathrm{~h}$, and $\gamma$-PGA yield was determined after the strain was incubated for $36 \mathrm{~h}$. The other conditions were seen in the "Material and methods" section. 
Water content in solid culture medium has a direct correlation with the water activity, which is a critical factor for microbial growth and metabolism under SSF. Poor water can limit some nutrients to be dissolved and transferred so as to inhibit microbial growth, while excessive water can inhibit the microbial growth by making substrate clustered and preventing air permeability and heat dissipation (Yadav et al. 2008). It's worth noting that $\gamma$-PGA has a capability of water retention to reduce the evaporation of the moist culture medium during incubation. On the other hand, water in the fluffy culture medium composed of rice husk could evaporate faster than that in the compact medium. Therefore, the optimization of water content in the culture medium is essential. Generally, the inoculum size has a dramatical effect on microbial biomass and metabolites synthesis. The effect of inoculum size (2-12\%) was explored on the co-production of NK and $\gamma$-PGA. Figure 6 indicated that the maximum yields of NK and $\gamma$-PGA were achieved with the inoculum sizes of 4 and $8 \%$, respectively. Excessive inoculum size could inhibit NK synthesis, but less inoculum size would not stimulate $\gamma$-PGA production completely. Accordingly, the inoculum size at $6 \%$ was chosen in further studies.

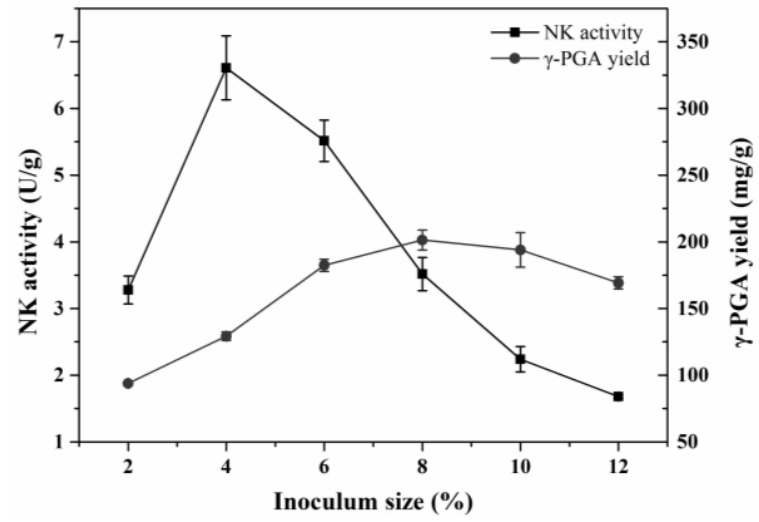

Figure 6 - Effect of inoculum size on NK and $\gamma$-PGA production.

In this experiment, the solid fermentation medium was composed of $30 \mathrm{~g}$ soybean flour and $18 \mathrm{~g}$ rice husk. Water content was determined as $70 \%$. NK activity was assayed after the strain was incubated for $24 \mathrm{~h}$, and $\gamma$-PGA yield was determined after the strain was incubated for $36 \mathrm{~h}$. The other conditions were seen in the "Material and methods" section.

Table 1 - Analysis of the difference of improved NK and $\gamma$-PGA production from the original ones along with reaction time.

\begin{tabular}{|c|c|c|c|c|c|c|c|c|c|}
\hline \multirow{2}{*}{$\begin{array}{l}\text { Reaction } \\
\text { time (h) }\end{array}$} & \multicolumn{5}{|c|}{ Improved } & \multicolumn{2}{|c|}{ Original } & \multirow[b]{2}{*}{$\mathbf{R}_{\mathrm{NK}}$} & \multirow[b]{2}{*}{$\mathbf{R}_{\mathbf{P G A}}$} \\
\hline & $\begin{array}{l}\mathbf{N K}_{\mathbf{s}} \\
(\mathbf{U} / \mathbf{g})\end{array}$ & $\begin{array}{c}\gamma-\mathbf{P G A}_{\mathrm{s}} \\
(\mathrm{mg} / \mathrm{g})\end{array}$ & $\mathrm{NK}_{\mathrm{p}}(\%)$ & $\begin{array}{c}\gamma-\text { PGA }_{\mathrm{p}} \\
(\%)\end{array}$ & $\mathbf{R}$ & $\begin{array}{c}\text { Top NK } \\
(\mathbf{U} / g)\end{array}$ & $\begin{array}{c}\text { Top } \gamma-P G A_{s} \\
(\mathrm{mg} / \mathrm{g})\end{array}$ & & \\
\hline 9 & 1.67 & 65.6 & 52.22 & 45.40 & 1.15 & & & 0.40 & 0.45 \\
\hline 18 & 3.14 & 126.2 & 98.58 & 87.34 & 1.13 & & & 0.75 & 0.87 \\
\hline 24 & 6.69 & 147.25 & 209.70 & 101.90 & 2.06 & 4.21 & & 1.59 & 1.02 \\
\hline 27 & 6.08 & 177.8 & 190.49 & 123.04 & 1.55 & & & 1.44 & 1.23 \\
\hline 36 & 3.70 & 222.6 & 116.13 & 154.05 & 0.75 & & 144.5 & 0.88 & 1.54 \\
\hline 45 & 3.57 & 194.8 & 111.95 & 134.81 & 0.83 & & & 0.85 & 1.35 \\
\hline 54 & 1.99 & 153 & 62.24 & 105.88 & 0.59 & & & 0.47 & 1.06 \\
\hline 63 & 1.13 & 127.6 & 35.51 & 88.30 & 0.40 & & & 0.27 & 0.88 \\
\hline 72 & 0.77 & 85.2 & 24.23 & 58.96 & 0.41 & & & 0.18 & 0.59 \\
\hline Mean & 3.19 & 144.45 & & & & & & & \\
\hline
\end{tabular}

$\mathrm{NK}_{\mathrm{s}}$ and $\gamma$ - $\mathrm{PGA}_{\mathrm{s}}$ denote the yields of $\mathrm{NK}$ and $\gamma$-PGA, respectively. $\mathrm{NK}_{\mathrm{p}}$ and $\gamma$ - $\mathrm{PGA}_{\mathrm{p}}$ are their percents relative to their corresponding means, respectively. R equals to the ratio of $\mathrm{NK}_{\mathrm{p}}$ to $\gamma-\mathrm{PGA}_{\mathrm{p}}$. Top $\mathrm{NK}_{\mathrm{s}}$ and Top $\gamma-\mathrm{PGA}_{\mathrm{s}}$ are the top yields of NK (incubated for $24 \mathrm{~h}$ ) and $\gamma$-PGA (incubated for $36 \mathrm{~h}$ ) before improvement, respectively. $\mathrm{R}_{\mathrm{NK}}$ and $\mathrm{R}_{\mathrm{PGA}}$ are the ratio of $\mathrm{NK}_{\mathrm{s}}$ to top $\mathrm{NK}_{\mathrm{s}}$ and the ratio of $\gamma-\mathrm{PGA}_{\mathrm{s}}$ to top $\gamma-\mathrm{PGA}_{\mathrm{s}}$, respectively. In the improved experiment, the solid fermentation medium was composed of $30 \mathrm{~g}$ soybean flour and $18 \mathrm{~g}$ rice husk. Inoculum size and water content were designed as $6 \%$ and $70 \%$, respectively. The other conditions were seen in the "Material and methods" section.

Inoculum size is very crucial for metabolite synthesis under SSF. Poor inoculum size can defer the synthetic progress of metabolite by prolonging the time course of microbial growth, while superfluous inoculum size can give rise to major nutrients of culture medium to be exhausted in microbial growth (Kashyap et al. 2002). Sufficient inoculation is beneficial to both the cell growth and product synthesis (Zeng et al. 2013). Accordingly, it is important for achieving a promising yield of metabolite to keep the balance between the biomass proliferating and available substrate(Pandey et al. 2000; Kashyap, et al. 2002). Rice husk and the small size effect of 
soybean have a positive effect on microbial distribution, and this reasonably accelerates the growth of microbial cell in this work. It was concluded that the inoculum size depended on not only the dispersability of culture medium but also microbial distribution.

B. subtilus natto was incubated in an optimized culture medium for 9-72 $\mathrm{h}$ based on the above results. It was found that NK and $\gamma$-PGA yields at 24-45 $\mathrm{h}$ were higher than their corresponding values at 9-72 h. NK and $\gamma$-PGA yields at 24 and $27 \mathrm{~h}$ surpassed the maximum ones without optimization. The ratio of $\mathrm{NK}_{\mathrm{p}}$ to $\gamma-\mathrm{PGA}_{\mathrm{p}}(\mathrm{R})$ at 27 $\mathrm{h}$ was closer to one relative to that at $24 \mathrm{~h}$ (Table 1). Consequently, the optimum incubation time was considered as $27 \mathrm{~h}$ for the co-production of NK and $\gamma$-PGA, where NK and $\gamma$-PGA yields were $6.08 \mathrm{U} / \mathrm{g}$ and $177.8 \mathrm{mg} / \mathrm{g}$, respectively, and the corresponding fibrinolytic activity of NK was 1564.4 IU/g (assayed by the standard fibrin plate method(Astrup and Müllertz 1952)). The fibrinolytic activity of NK and $\gamma$-PGA yield was converted to $2503.4 \mathrm{IU} / \mathrm{g}$ for soybean (IU/gs) and $320 \mathrm{mg} / \mathrm{gs}$, increasing by approximate 7 and 2-fold factors relative to their original yields (342.98 $\mathrm{IU} / \mathrm{gs}$ and $144.5 \mathrm{mg} / \mathrm{gs}$ ), respectively, when soybean instead of total solid supports was regarded as a measurement baseline. Furthermore, the $\gamma$-PGA yield in this work was higher than that $(51.3 \mathrm{mg} / \mathrm{g})$ in co-production of iturin A and $\gamma$ PGA (Yao et al. 2012). Also, the NK and $\gamma$-PGA yields were higher than the ones reported by Zeng et al.(2013). Troublesomely, it is difficult to extract NK from the co-production ferment including $\gamma$-PGA because high concentration of $\gamma$ PGA could disturb NK separation (Wei et al. 2012). Microbial $\gamma$-PGA with relatively high molecular weight can make the ferments too viscous to be separated easily. Therefore, it is very necessary to construct an efficient method for the departure of NK from $\gamma$-PGA in the further work.

\section{CONCLUSIONS}

In summary, the smaller size of the substrate particles significantly improved the co-production of NK and $\gamma$-PGA by B. subtilus natto. Rice husk enhanced the soybean utilization and decreased the detrimental effect of the smaller size of the substrate particles related to aeration. The fermented solid medium could be utilized to prepare organic fertilizer. These findings suggested that the co-production of $\mathrm{NK}$ and $\gamma$ PGA under SSF with agro-residues as substrate could be considered as an efficient method to exploit agro-residues for economical production of higher-value products.

\section{ACKNOWLEDGMENTS}

The authors gratefully acknowledge financial support granted to this work by National Natural Science Foundation of China (51202002), Key Project of Provincial Natural Science Research of Colleges and Universities in Anhui Province (KJ2014A025), and National Training Programs of Innovation and Entrepreneurship for Undergraduates (201210363042, 201310363076).

\section{REFERENCES}

Astrup T, Müllertz S. The fibrin plate method for estimating fibrinolytic activity. Arch Biochem Biophys. 1952; 40:346-351.

Bajaj I, Lele S, Singhal R. Enhanced production of poly $(\gamma$-glutamic acid) from Bacillus licheniformis NCIM 2324 in solid state fermentation. J Ind Microbiol Biot. 2008; 35:1581-1586.

Beniwal V, Goel G, Kumar A, Chhokar V. Production of tannase through solid state fermentation using Indian Rosewood (Dalbergia Sissoo) sawdust - a timber industry waste. Ann Microbiol. 2013; 63:583-590.

Chen X, Chen S, Sun M, Yu Z. High yield of poly- $\gamma$ glutamic acid from Bacillus subtilis by solid-state fermentation using swine manure as the basis of a solid substrate. Bioresource Technol. 2005; 96:18721879.

Deepak V, Kalishwaralal K, Ramkumarpandian S, Babu SV, Senthilkumar S, Sangiliyandi G. Optimization of media composition for nattokinase production by Bacillus subtilis using response surface methodology. Bioresource Technol. 2008; 99:8170-8174.

Fujita M, Hong K, Ito Y, Misawa S, Takeuchi N, Kariya $\mathrm{K}$, et al. Transport of nattokinase across the rat intestinal tract. Biol Pharm Bull. 1995; 18:1194-1196.

Goto A, Kunioka M. Biosynthesis and hydrolysis of poly (glutamic acid) from Bacillus subtilis IFO3335. Biosci Biotechnol Biochem. 1992; 56:1031-1035.

Jana A, Maity C, Halder SK, Mondal KC, Pati BR, Mohapatra PKD. Tannase production by Penicillium purpurogenum PAF6 in solid state fermentation of tannin-rich plant residues following OVAT and RSM. Appl Biochem Biotechnol. 2012; 167:1254-1269. 
Kashyap P, Sabu A, Pandey A, Szakacs G, Soccol CR. Extracellular 1-glutaminase production by Zygosaccharomyces rouxii under solid-state fermentation. Process Biochem. 2002; 38:307-312.

Pandey A Solid-state fermentation. Biochem Eng J. 2003; 13:81-84.

Pandey A, Soccol CR, Mitchell D. New developments in solid state fermentation: I-bioprocesses and products. Process Biochem. 2000; 35:1153-1169.

Richard A, Margaritis A. Rheology, oxygen transfer and molecular weight characteristics of poly(glutamic acid) fermentation by Bacillus subtilis. Biotech Bioeng. 2003; 82:299-305.

Selwal MK, Selwal KK. High-level tannase production by Penicillium atramentosum KM using agro residues under submerged fermentation. Ann Microbiol. 2012; 62:139-148.

Shih IL, Van YT. The production of poly- $(\gamma$-glutamic acid) from microorganisms and its various applications. Bioresource Technol. 2001; 79:207-225.

Sumi H, Nakajima N, Mihara H. In vitro and in vivo fibrinolytic properties of nattokinase. Thromb Haemosts. 1992; 89:1267.

Wang F, Hui N, Cai HN, Xiao AF. Tea stalks-a novel agro-residue for the production of tannase under solid state fermentation by Aspergillus niger JMU-TS528. Ann Microbiol. 2013; 63:897-904.

Wang QJ, Chen SW, Zhang JB, Sun M, Liu ZD, Yu ZN. Co-producing lipopeptides and poly- $\gamma$-glutamic acid by solid-state fermentation of Bacillus subtilis using soybean and sweet potato residues and its biocontrol and fertilizer synergistic effects. Bioresource Technol. 2008; 99:3318-3323.

Wei H, Zhao XY, J LJ. The detecting method of nattokinase thrombolysis activity. JShandong Inst Light ind (in Chinese). 2007; 21:60-63.
Wei XT, Luo MF, Xie YC, Yang LR, Li HJ, Xu L, et al. Strain screening, fermentation, separation, and encapsulation for production of nattokinase functional food. Appl Biochem Biotechnol. 2012; 168:1753-1764.

Yadav A, Aggarwal N, Kumar K, Kumar A. Tannase production by Aspergillus fumigatus MA under solidstate fermentation. World J Microb Biot. 2008; 24:3023-3030.

Yao DH, Ji ZX, Wang CJ, Qi GF, Zhang LL, Ma X, et al. Co-producing iturin $\mathrm{A}$ and poly- $\gamma$-glutamic acid from rapeseed meal under solid state fermentation by the newly isolated Bacillus subtilis strain 3-10. World J Microb Biot. 2012; 28:985-991.

Zeng W, Chen GG, Zhang YK, Wu KY, Liang ZQ. Studies on the UV spectrum of poly ( $\gamma$-glutamic acid) based on development of a simple quantitative method. Int J Biol Macromol. 2012; 51:83-90.

Zeng W, Li W, Shu L, Yi JY, Chen GG, Liang ZQ. Nonsterilized fermentative co-production of poly $(\gamma$ glutamic acid) and fibrinolytic enzyme by a thermophilic Bacillus subtilis GXA-28. Bioresource Technol. 2013; 142:697-700.

Zheng Z, Zuo Z, Liu Z, Tsai K, Liu A, Zou G. Construction of a 3D model of nattokinase, a novel fibrinolytic enzyme from Bacillus natto: A novel nucleophilic catalytic mechanism for nattokinase. $J$ Mol Graph Model. 2005; 23:373-380.

Zhu AT, Jiang YW, Xie GS, Chen SW. Effects of exogenous poly- $\gamma$-glutamic acid on drought tolerance and osmotic adjustment of rice seedlings. $\mathrm{J} \mathrm{Nucl}$ Agric Sci (in Chinese). 2010; 24:1269-1273.

Received: April 02, 2015; Accepted: May 25, 2015. 